\title{
Authors Reply
}

\section{Anopheles control is considerably more complicated than Aedes control}

\author{
Djane Clarys Baia-da-Silva ${ }^{[1],[2], ~ G i s e l y ~ C a r d o s o ~ d e ~ M e l o[1],[2], ~ P a u l o ~ P i m e n t a ~}{ }^{[1],[2],[3],}$ \\ Marcus Vinícius Guimarães de Lacerda ${ }^{[1],[2],[4]}$ and Wuelton Marcelo Monteiro[1],[2]
}

\author{
[1]. Fundação de Medicina Tropical Dr. Heitor Vieira Dourado, Manaus, AM, Brasil. \\ [2]. Universidade do Estado do Amazonas, Programa de Pós-Graduação em Medicina Tropical, Manaus, AM, Brasil. \\ [3]. Fundação Oswaldo Cruz, Instituto de Pesquisas René Rachou, Belo Horizonte, MG, Brasil. \\ [4]. Fundação Oswaldo Cruz, Instituto de Pesquisas Leônidas and Maria Deane, Manaus, AM, Brasil.
}

\section{Dear Editor:}

The letter by Dr. Wermelinger ${ }^{1}$ doi: 10.1590/0037-8682-0385-2019 provides an interesting critique of the evolution of vector-borne disease control in Brazil, highlighting the need to invest in new environment-friendly control methods that are economically viable and feasible to implement.

In terms of innovative methods for vector control involving interaction between mosquitoes and bacteria, greater advances have been made in controlling dengue than malaria due to the differing biology of the respective vectors.

Previous studies on the anopheline microbiome indicate that there is no natural obligate endosymbiont in the Anopheles genus, which is in contrast to what has been observed in some Aedes species that are susceptible to Wolbachia. This susceptibility, along with the fact that colonized mosquitoes are refractory to dengue virus infection, represents an exciting potential new form of biocontrol for arboviral diseases, including dengue. Strains of Wolbachia, deliberately introduced into Ae. aegypti mosquitoes, have been shown a capacity to spread in high frequencies within mosquito populations in release trials, and mosquitoes infected with these strains have shown markedly reduced vector competence ${ }^{2,3,4}$. In Anopheles, it is possible that secondary symbionts may have become recently more susceptible to anophelines and are, therefore, not obligate ${ }^{5}$, although they may fulfill a role in host biology and susceptibility to Plasmodium ${ }^{6}$.

\footnotetext{
Corresponding author: Wuelton Marcelo Monteiro.

e-mail: wueltonmm@gmail.com

(1) 0000-0002-0848-1940

Received 8 September 2019

Accepted 11 October 2019
}

Wermelinger's letter also highlights the need to consider methodological principles that should guide control interventions, with an emphasis on integrated vector management. This appears possible using Wolbachia in Ae aegypti since Wolbachia also blocks other circulating arboviruses such as Chikungunya and Zika viruses ${ }^{6,7}$. Regarding Anopheles, only Plasmodium parasites are transmitted, making it difficult to plan joint control strategies involving other diseases.

Ae. aegypti is an extremely anthropophilic mosquito, frequently found in urban areas, that lives in or around households or other buildings frequented by people, such as businesses and schools. An. darlingi, the most important vector of malaria in Brazil, is the most frequent anopheline found within human housing. This mosquito is particularly aggressive towards humans, usually attacking people inside their homes in the early evening. Ae. aegypti mosquitoes prefer to breed in areas such as stagnant water and in artificial containers such as flower vases, uncovered barrels, buckets, and discarded tires, whereas An. darlingi use natural and artificial water bodies, such as ponds, riverbanks, puddles, and water-logged fields, preferably involving clean water comprising organic matter, aquatic vegetation, and shade. Therefore, anophelines are much more exposed to other environmental bacteria that could compete with Wolbachia. In addition, malaria also occurs in rural, riverine, and indigenous areas, where the maintenance of engineered vector populations may only last for short periods of time.

All these factors are likely to help explain studies indicating the usefulness of artificially manipulated microbiome mosquitoes in relation to dengue fever and the absence of similar field studies concerning malaria. Recently, the Brazilian Ministry of Health, in partnership with Fiocruz's World Mosquito Program-Brazil, as part of a campaign against dengue, Zika, Chikungunya, and yellow fever, announced the expansion of the Wolbachia method into three Brazilian cities, Campo Grande (MS), Belo Horizonte (MG), and 
Petrolina $(\mathrm{PE})^{8}$. This is a field where malaria researchers are only in the early stages of basic research; further studies are needed to achieve a breakthrough.

Currently, promising entomopathogens remain restricted to Bacillus thuringiensis and B. sphaericus. Even for the available strains, information concerning their action in the field is scarce in the Amazonia ${ }^{9}$, but it indicates that these biolarvicides may be effective for larval source management aiming at malaria control ${ }^{9,10}$. Furthermore, dengue has overtaken malaria in terms of the volume of research concerning this issue. A PubMed search (accessed on August 28,2019) using the terms Bacillus thuringiensis OR Bacillus sphaericus and Aedes OR dengue retrieved 676 publications, whereas using the terms Bacillus thuringiensis OR Bacillus sphaericus and Anopheles OR malaria identified 384 publications.

To reduce malaria transmission, a successful programmer needs to focus on several aspects, including early diagnosis, use of effective antimalarial drugs, and vector control ${ }^{11}$. These strategies are critical for malaria elimination. In this context, tools such as genetically modified mosquitos, transmission-blocking strategies (using drugs, antibodies, or even Wolbachia-like microorganisms), and breeding site management with bioinsecticides should be considered. However, apart from the unpublished experiences of individual malaria control programmers and unlike the situation with dengue, there is a lack of robust evidence supporting the efficacy of specific tools for malaria control and elimination through employing vector control measures.

\section{AUTHORS' CONTRIBUTION}

All authors contributed to the writing and review.

\section{CONFLICT OF INTEREST}

The authors declare that there is no conflict of interest.

\section{REFERENCES}

1. Wermelinger ED. Reflections on vector control in Brazil. Rev Soc Bras Med Trop. 2020;53: e20190385.

2. Hoffmann AA, Montgomery BL, Popovici J, Iturbe-Ormaetxe I, Johnson PH, Muzzi F, et al. Successful establishment of Wolbachia in Aedes populations to suppress dengue transmission. Nature 2011;476(7361):454-7.

3. Ferguson NM, Kien DTH, Clapham H, Aguas R, Trung VT, Chau TNB, et al. Modeling the impact on virus transmission of Wolbachia-mediated blocking of dengue virus infection of Aedes aegypti. Sci Transl Med. 2015;7(279):279ra37.

4. Dorigatti I, McCormack C, Nedjati-Gilani G, Ferguson NM. Using Wolbachia for Dengue Control: Insights from Modelling. Trends Parasitol. 2018;34(2):102-13.

5. Villegas LM, Pimenta PFP. Metagenomics, paratransgenesis and the Anopheles microbiome: A portrait of the geographical distribution of the anopheline microbiota based on a meta-analysis of reported taxa. Mem Inst Oswaldo Cruz. 2014;109(5):672-84.

6. Moreira LA, Iturbe-Ormaetxe I, Jeffery JA, Lu G, Pyke AT, Hedges LM, et al. A Wolbachia Symbiont in Aedes aegypti Limits Infection with Dengue, Chikungunya, and Plasmodium. Cell. 2009;139(7):1268-78.

7. Dutra HLC, Rocha MN, Dias FBS, Mansur SB, Caragata EP, Moreira LA. Wolbachia Blocks Currently Circulating Zika Virus Isolates in Brazilian Aedes aegypti Mosquitoes. Cell Host Microbe. 2016;19(6):771-4.

8. Ministério da Saúde anuncia expansão do método Wolbachia. 2019. https://pesquisasaude.saude.gov.br/noticiaLerMais.xhtml?id=1171.

9. Rodrigues IB, Tadei WP, Da Silva Dias JMC. Larvicidal activity of Bacillus sphaericus 2362 against Anopheles nuneztovari, Anopheles darlingi and Anopheles braziliensis (Diptera, Culicidae). Rev Inst Med Trop Sao Paulo. 1999;41(2):101-5.

10. Rodrigues IB. Atividade Larvicida de Bacillus sphaericus 2362 contra Anopheles sp. (Diptera, Culicidae) em rios do Amazonas, Brasil. BioAssay. 2013;8(0).

11. Baia-da-Silva DC, Brito-Sousa JD, Rodovalho SR, Peterka C, Moresco G, Lapouble OMM, et al. Current vector control challenges in the fight against malaria in Brazil. Rev Soc Bras Med Trop. 2019;52:e20180542. 